\title{
Glaucoma bilateral por fechamento angular induzido por sulfametoxazol-trimetoprima: relato de caso
}

\author{
Bilateral angle-closure glaucoma induced by trimetoprim and sulfamethoxazole \\ combination:case report
}

\author{
Viviane Souto Spadoni ${ }^{1}$ \\ Melissa Manfroi Dal Pizzol ${ }^{2}$ \\ Carlos Henrique Gervini Muniz ${ }^{3}$ \\ Jacobo Melamed ${ }^{4}$ \\ João Borges Fortes Filho
}

Trabalho realizado no Serviço de Oftalmologia do Hospital de Clínicas de Porto Alegre - HCPA - Porto Alegre (RS) - Brasil.

Residente do Serviço de Oftalmologia do Hospital de Clínicas de Porto Alegre - HCPA - Porto Alegre (RS) - Brasil.

${ }^{2}$ Residente do Serviço de Oftalmologia do HCPA - Porto Alegre (RS) - Brasil.

Residente do Serviço de Oftalmologia do HCPA - Porto Alegre (RS) - Brasil.

${ }^{4}$ Professor de Oftalmologia da Faculdade de Medicina da Universidade Federal do Rio Grande do Sul - UFRGS - Porto Alegre (RS) - Brasil; Chefe do Setor de Uveítes do Serviço de Oftalmologia do HCPA - Porto Alegre (RS) - Brasil.

Professor de Oftalmologia da Faculdade de Medicina da UFRGS - Porto Alegre (RS) - Brasil.

Endereço para correspondência: Viviane Souto Spadoni. Rua Luzitana, 1188/302 - Porto Alegre (RS) CEP 90520-080

E-mail: vspadoni@terra.com.br

Recebido para publicação em 09.04.2006

Última versão recebida em 16.10.2006

Aprovação em 25.10.2006

Nota Editorial: Depois de concluída a análise do artigo sob sigilo editorial e com a anuência da Dra. Maria Rosa Bet de Moraes e Silva sobre a divulgação de seu nome como revisora, agradecemos sua participação neste processo.

\begin{tabular}{|l|}
\hline RESUMO \\
\hline Sulfametoxazol e trimetoprima (cotrimoxazol) é uma combinação de dro- \\
gas amplamente usada no tratamento e profilaxia de inúmeras infecções \\
sistêmicas. Esta droga e outras derivadas da sulfa podem causar uma \\
síndrome ocular rara caracterizada por efusão coroidal supracililar com \\
miopização transitória e glaucoma por fechamento angular. A maioria dos \\
autores atribui o glaucoma ao edema do corpo ciliar que leva ao deslo- \\
camento anterior do diafragma irido-cristaliniano causando fechamento \\
do ângulo camerular. Este trabalho descreve um caso raro no qual a \\
síndrome ocorreu após o uso desta combinação de drogas e evoluiu para \\
um desfecho desfavorável. Paciente de 49 anos, sexo masculino, branco \\
com diagnóstico de síndrome da imunodeficiência adquirida iniciou \\
tratamento profilático para Pneumocystis carinii com cotrimoxazol. Qua- \\
tro dias após, apresentou quadro de dor ocular, hiperemia e quemose \\
conjuntival, glaucoma agudo por fechamento angular com pressões \\
intra-oculares maiores que 50 mmHg e efusão coroidal 360 ${ }^{\circ}$, com os \\
achados presentes nos dois olhos. Nesse mesmo dia, a medicação foi \\
suspensa com diminuição da pressão intra-ocular após quatro dias. O \\
paciente evoluiu com catarata total e phthisis bulbi bilateral nos dois \\
meses subseqüentes. Os casos já descritos mencionam a melhora clínica \\
completa do quadro ocular após a suspensão da medicação. Este seria \\
o primeiro caso na literatura no qual a evolução foi desfavorável apesar \\
do diagnóstico e da suspensão precoce da medicação causadora.
\end{tabular}

Descritores: Glaucoma; Glaucoma de ângulo fechado/induzido quimicamente; Combinação trimetoprima-sulfametoxazol/efeitos adversos; Relatos de casos [Tipo de publicação]

\section{INTRODUCÃO}

Glaucoma bilateral por fechamento angular (GFA) e miopia transitória induzidos por drogas vêm sendo descritos como efeitos adversos raros e idiossincráticos de drogas sistêmicas contendo sulfonamidas, tais como: diuréticos, antibióticos e antidepressivos ${ }^{(1-2)}$. Miopia aguda como reação às sulfonamidas foi descrita primeiramente em $1938^{(3)}$.

Sulfametoxazol e trimetoprima (cotrimoxazol) é uma combinação de antibióticos amplamente usada no tratamento e profilaxia de inúmeras infecções. Apresenta como efeito adverso raro, uma síndrome caracterizada por miopização transitória e GFA. Esse processo é causado pela efusão coroidal supracililar que gera alterações irido-cristalinianas levando à anteriorização do cristalino e corpo ciliar ${ }^{(4)}$. Todos os casos relatados na literatura evoluíram para a resolução completa dessa condição após a descontinuação precoce da droga. 
O objetivo deste trabalho é relatar o primeiro caso de GFA bilateral com efusão coroidal supraciliar total após o uso de cotrimoxazol com evolução clínica desfavorável apesar do diagnóstico e suspensão precoce da medicação.

\section{RELATO DO CASO}

Paciente de 49 anos, sexo masculino, branco, consultou na emergência do Hospital de Clínicas de Porto Alegre (HCPA) com história de confusão e desorientação há três dias. Em relação à história médica pregressa, apresentava diagnóstico de síndrome da imunodeficiência adquirida e hipertensão arterial sistêmica para as quais não fazia uso de nenhuma medicação. Foi indicada internação para investigação diagnóstica e iniciado tratamento profilático para Pneumocystis carinii com cotrimoxazol.

Quatro dias após iniciada profilaxia, paciente apresentou quadro de dor ocular importante, hiperemia e quemose bilaterais, sendo solicitada avaliação oftalmológica.

Ao exame oftalmológico, o paciente não informava acuidade visual devido ao quadro neurológico. Na biomicroscopia observavam-se midríase média fixa, edema microcístico e estromal de córnea, câmara anterior rasa com toque iridocorneano periférico (Figura 1), quemose conjuntival importante e edema palpebral em ambos os olhos (AO). A pressão intra-ocular (PIO) obtida pelo tonômetro de Perkins era maior que $50 \mathrm{mmHg}$ em $\mathrm{AO}$. A fundoscopia e retinoscopia não foram possíveis devido à opacidade cristaliniana e edema de córnea.

Foi feito o diagnóstico de GFA bilateral e iniciado tratamento com acetazolamida $250 \mathrm{mg}$ via oral, e manitol $20 \% 250 \mathrm{ml}$ endovenoso, além de colírios de maleato de timolol 0,5\%,

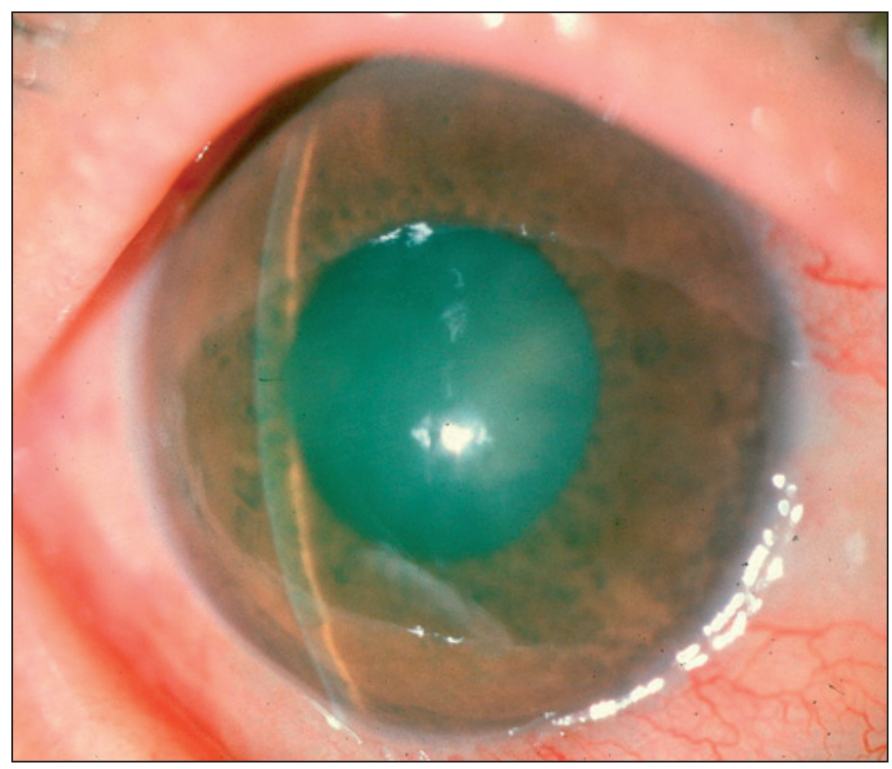

Figura 1-Biomicroscopia do olho esquerdo mostrando edema corneano, midríase média, câmara anterior rasa com toque irido-corneano periférico tartarato de brimonidina $0,2 \%$, pilocarpina $2 \%$. Após uma hora do início do tratamento, a PIO reduziu para $38 \mathrm{mmHg}$ no olho direito (OD) e $42 \mathrm{mmHg}$ no olho esquerdo (OE).

Nesse mesmo dia, foi realizada ecografia ocular que demonstrou efusão coroidal $360^{\circ}$ com descolamento de coróide periférico em AO (Figura 2). As medidas da profundidade da
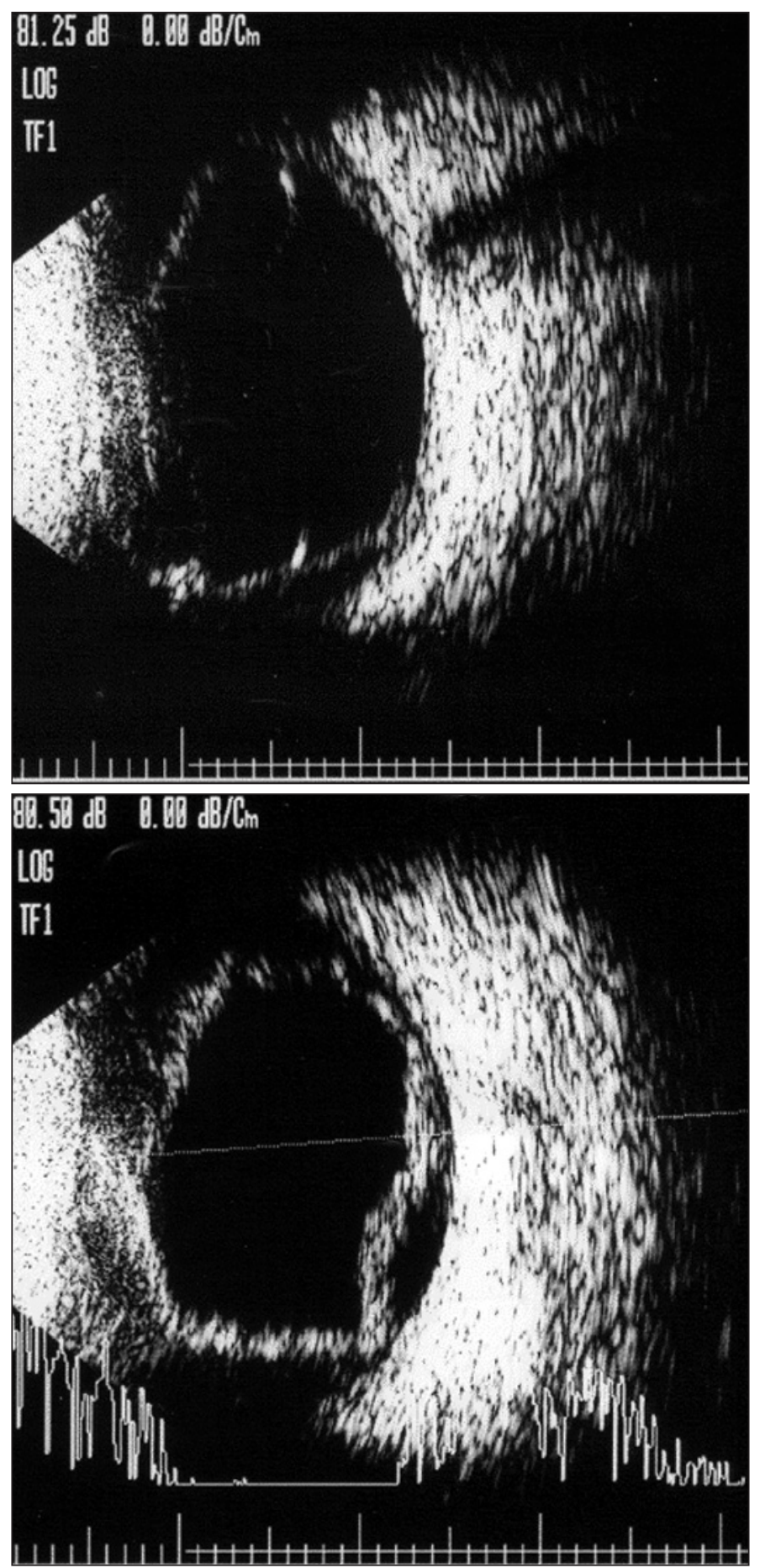

Figura 2 - Ecografia mostrando efusão coroidal supraciliar $360^{\circ}$ e descolamento de coróide periférico no OD e OE, respectivamente 
câmara anterior, espessura cristaliniana e cavidade vítrea do OD eram 1,6 mm, 5,4 mm e 15,2 mm respectivamente e apresentavam-se similares no OE.

Com base na história clínica e exame físico, foi cogitada a hipótese diagnóstica de efusão coroidal supracililar secundária ao uso de sulfametoxazol, sendo então suspensa esta medicação quatro dias após iniciada.

No dia seguinte, foram ainda suspensos o colírio de pilocarpina e acetazolamida, visto que esta também contem sulfa. Foram também iniciados corticóides tópico e oral com o objetivo de reduzir a resposta inflamatória. A tabela 1 mostra as drogas utilizadas durante a evolução do caso.

O quadro permaneceu inalterado até quatro dias após a suspensão da medicação, quando a PIO havia diminuído para 10 mmHg em AO e, ao exame, observava-se catarata e câmara anterior profunda (Figura 3) e a ecografia mostrava melhora discreta da efusão coroidal. Nos dias subseqüentes a PIO foi diminuindo até zero em AO, permanecendo uma pequena efusão coroidal supraciliar.

O paciente permaneceu sendo avaliado a cada três dias durante a internação que se prolongou por dois meses após a data do primeiro exame, sem melhora do quadro neurológico. No momento da alta hospitalar, apresentava catarata total intumescente bilateral, pressão intra-ocular de zero e phthisis bulbi bilateral.

\section{DISCUSSÃO}

A síndrome da efusão coroidal supracililar induzida por drogas ocorre raramente e pode ser causada por drogas derivadas da sulfa, incluindo acetazolamida, cotrimoxazol ${ }^{(5)}$, hidroclorotiazida, trianterene ${ }^{(6)}$ e topiramato ${ }^{(7-9)}$.

O mecanismo primário que leva a essa síndrome permanece desconhecido, mas é sugerido que este esteja relacionado à inibição da anidrase carbônica ou a um efeito mediado por prostaglandinas ${ }^{(10)}$. Embora exista controvérsia, a maioria dos autores atribui o glaucoma ao edema do corpo ciliar que leva ao deslocamento anterior do diafragma irido-cristaliniano, causando fechamento do ângulo camerular ${ }^{(7,11)}$. Ramos-Esteban et al. relatam um caso de efusão supraciliar bilateral após o uso de cotrimoxazol acompanhado com auxílio da biomicros- copia ultra-sônica (UBM) onde os achados corroboraram para esse mecanismo fisiopatogênico proposto ${ }^{(5)}$.

Como o mecanismo de fechamento angular não envolve bloqueio pupilar, iridectomias periféricas e mióticos são inúteis no tratamento desse tipo de glaucoma ${ }^{(4,7-8)}$. Devido a esses fatores, procedeu-se, no caso aqui relatado, a descontinuação da pilocarpina e a não realização de iridotomias periféricas.

Drogas inibidoras da produção do humor aquoso vêm sendo implicadas na etiopatogenia da hipotonia e efusão coroidal, no entanto, a literatura associa essa ocorrência a pacientes submetidos à cirurgia filtrante e/ou cirurgia de catarata nos quais faziam uso crônico de maleato de timolol e/ou acetazolamida $^{(12-14)}$. No caso relatado, o paciente não tinha história prévia de uso de drogas anti-glaucomatosas e/ou cirurgia intra-ocular, além de ter sido utilizado drogas inibidoras da produção do aquoso por curto período, sendo, portanto, improváveis causadores do quadro clínico relatado.

Todos os relatos da literatura são unânimes quanto à evolu-

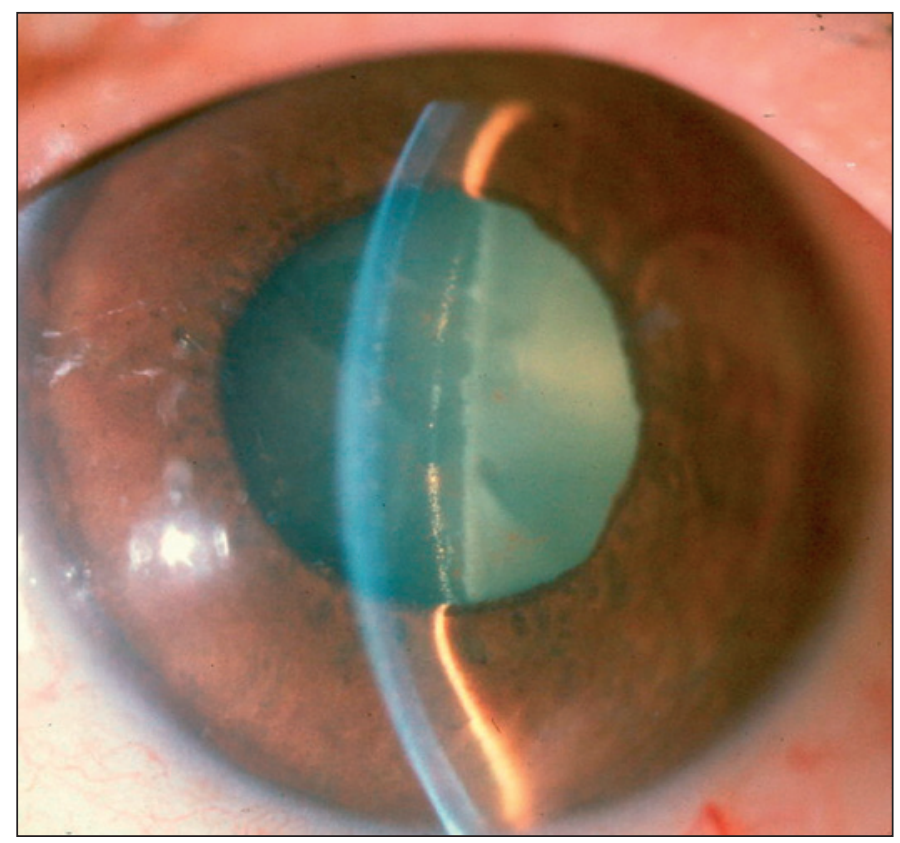

Figura 3 - Biomicroscopia do olho esquerdo após a suspensão da medicação, evidenciando catarata e a câmara anterior profunda

\begin{tabular}{|c|c|c|c|c|c|}
\hline $1^{\circ} \mathrm{dia}$ & $4^{\circ} \mathrm{dia}$ & $5^{\circ} \mathrm{di}$ & $6^{\circ} \mathrm{di}$ & $7^{\circ} \mathrm{di}$ & $8^{\circ} \mathrm{di}$ \\
\hline \multicolumn{6}{|c|}{ Clotrimoxazol } \\
\hline & Acetazolamida & & & & \\
\hline & Pilocarpina & & & & \\
\hline & $\begin{array}{l}\text { Tartarato de } \\
\text { brimonidina }\end{array}$ & $\begin{array}{l}\text { Tartarato de } \\
\text { brimonidina }\end{array}$ & $\begin{array}{l}\text { Tartarato de } \\
\text { brimonidina }\end{array}$ & $\begin{array}{l}\text { Tartarato de } \\
\text { brimonidina }\end{array}$ & \\
\hline & Manitol & Manitol & Manitol & Manitol & \\
\hline & Timolol & Timolol & Timolol & Timolol & \\
\hline & & Dexametasona $1 \%$ & Dexametasona $1 \%$ & Dexametasona $1 \%$ & Dexametasona $1 \%$ \\
\hline & & Prednisona $60 \mathrm{mg}$ & Prednisona $60 \mathrm{mg}$ & Prednisona $60 \mathrm{mg}$ & Prednisona $60 \mathrm{mg}$ \\
\hline
\end{tabular}


ção clínica favorável após a suspensão imediata da medicação derivada da sulfa. No entanto, apresentamos um caso onde o paciente, apesar da melhora da PIO e da amplitude da câmara anterior, evoluiu para phthisis bulbi bilateral. Postulamos que isso seja atribuído a uma falência aguda e permanente do corpo ciliar, visto que a PIO se apresentava muito elevada (maior que $50 \mathrm{mmHg}$ em AO) no momento do diagnóstico, diferentemente das pressões mais baixas relatadas na literatura.

Este é o primeiro caso relatado de evolução clínica desfavorável apesar do diagnóstico precoce e da suspensão imediata da medicação. Os autores sugerem que a patologia imunológica do paciente e a gravidade da apresentação do quadro possam ter contribuído para esse desfecho.

É importante salientar que frente a um paciente com GFA bilateral e história de tratamento com medicação derivada da sulfa, o oftalmologista deve sempre suspeitar dessa potencial complicação e imediatamente indicar a retirada da droga, visto que, na maioria dos casos, a remissão completa do quadro pode ocorrer com a descontinuação da medicação.

\section{ABSTRACT}

Sulfamethoxazole-trimethoprim (cotrimoxazole) is an antibiotic combination widely used for infections treatment and prophylaxis. These and others sulfonamides have been implicated in a rare syndrome of choroidal effusion with transient myopia and angle-closure glaucoma. Previous cases reported in literature evolved to complete resolution after drug withdrawal. In contrast, we describe a rare case in which a patient developed the syndrome while taking cotrimoxazole, but did not recover visual acuity. A 49-year-old man started Pneumocystis carini prophylaxis with cotrimoxazole; four days later, the patient presented severe ocular pain, hyperemia and chemosis. Intraocular pressure reached more than $50 \mathrm{mmHg}$ in both eyes a $360^{\circ}$ choroidal effusion occurred. Medication was removed soon after the diagnosis was suspected and intraocular pressure decreased in four days. Even so total cataract and phthisis bulbi occurred in both eyes two months later. This would be the first case in the literature in which the outcome was unfavorable despite early diagnosis and withdrawal of the drug.

Keywords: Glaucoma; Glaucoma, angle-closure/chemically induced; Trimethoprim-sulfamethoxazole combination/adverse effects; Case reports [Publication type]

\section{REFERÊNCIAS}

1. Grant WM, Schuman JS. Toxicology of the eye. 4th ed. Springfield: Charles C Thomas; 1993. p.22-4.

2. Ikeda N, Ikeda T, Nagata M, Mimura O. Ciliochoroidal effusion syndrome induced by sulfa derivatives. Arch Ophthalmol. 2002;120(12):1775.

3. Berns W. Proceedings of the $3^{\text {rd }}$ Meeting of the Swedish Ophthalmological Society. December, 1938. Acta Ophthalmol. 1940;18:96-8.

4. Postel EA, Assalian A, Epstein DL. Drug-induced transient myopia and angleclosure glaucoma associated with supraciliary choroidal effusion. Am J Ophthalmol. 1996;122(1):110-2.

5. Ramos-Esteban JC, Goldberg S, Danias J. Drug induced acute myopia with supraciliary choroidal effusion in a patient with Wegener's granulomatosis. $\mathrm{Br}$ J Ophthalmol. 2002;86(5):594-6.

6. Soylev MF, Green RL, Feldon SE. Choroidal effusion as a mechanism for transient myopia induced by hydrochlorothiazide and triamterene. Am J Ophthalmol. 1995;120(3):395-7.

7. Banta JT, Hoffman K, Budenz DL, Ceballos E, Greenfield DS. Presumed topiramate-induced bilateral acute angle-closure glaucoma. Am J Ophthalmol. 2001; 132(1):112-4.

8. Rhee DJ, Goldberg MJ, Parrish RK. Bilateral angle-closure glaucoma and ciliary body swelling from topiramate. Arch Ophthalmol. 2001;119(11):1721-3.

9. Sankar PS, Pasquale LR, Grosskreutz CL Uveal effusion and secondary angleclosure glaucoma associated with topiramate use. Arch Ophthalmol. 2001;119 (8):1210-1.

10. Krieg PH, Shipper I. Drug-induced ciliary body oedema: a new theory. Eye. 1996;10(Pt 1):121-6.

11. Bovino JA, Marcus DF. The mechanism of transient myopia induced by sulfonamide therapy. Am J Ophthalmol. 1982;94(1):99-102.

12. Vela MA, Campbell DG. Hypotony and ciliochoroidal detachment following pharmacologic aqueous suppressant therapy in previously filtered patients. Ophthalmology. 1985;92(1):50-7.

13. Geyer O, Neudorfer M, Lazar M. Recurrent choroidal detachment following timolol therapy in previously filtered eye. Choroidal detachment post filtering surgery. Acta Ophthalmol (Copenh). 1992;70(5):702-3.

14. Rachmiel R, Pollack A, Oliver M, Zalish M. Recurrent choroidal detachment following combined trabeculectomy and cataract extraction. Ophthalmic Surg Lasers. 1996;27(9):795-7.

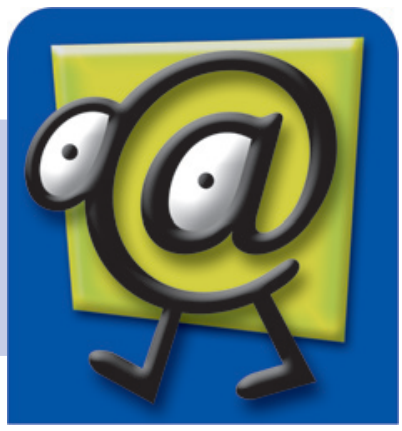

